\title{
Optimum Sleep is Important for Reducing the Risk of Cardiovascular Mortality \& Morbidity
}

\section{Abstract}

Cardiovascular disease (CVD) is the leading cause of death worldwide. Humans spend up to a third of their life asleep, yet sleep problems are common in society [1]. Sleep disorders, such as insomnia and obstructive sleep apnoea, not only affect optimal functioning and productivity, but have long term impacts on health. Over the last two decades, emerging research has linked sleep duration, quality and sleep disorders with CVD, metabolic syndrome and obesity. This essay will explore the role of sleep in maintaining a healthy heart, and how strategies to improve sleep can reduce CVD risk.

Key words

Sleep; sleep deprivation; cardiovascular disease; obesity

\section{Sleep Duration and Mortality}

Too little or too much sleep is associated with an increase in mortality. A meta-analysis showed that sleep duration and all-cause mortality were associated in a U-shaped curve, where the lowest risk was in those who slept between 7 to 8 hours per night [2,3]. After review of 864 published articles, the following sleep durations were recommended by the American Academy of Sleep Medicine: [4]

- Infants 4 months to 12 months should sleep 12 to 16 hours per 24 hours (including naps)

- Children 1 to 2 years of age should sleep 11 to 14 hours per 24 hours (including naps)

- Children 3 to 5 years of age should sleep 10 to 13 hours per 24 hours (including naps)

- Children 6 to 12 years of age should sleep 9 to 12 hours per 24 hours

- Teenagers 13 to 18 years of age should sleep 8 to 10 hours per 24 hours

Sleeping the number of recommended hours on a regular basis is associated with better health outcomes including: improved attention, behaviour, learning, memory, emotional regulation, quality of life, and mental and physical health. Regularly sleeping fewer than the number of recommended hours is associated with attention, behaviour, and learning problems. Insufficient sleep also increases the risk of accidents, injuries, hypertension, obesity, diabetes, and depression.
Triya A Chakravorty BA (Oxon)

Oxford Medical School, Queen's College, University of Oxford, UK

Triya.chakravorty@queens.ox.ac. $\underline{\text { uk }}$

Cite as: Chakravorty, T.A. (2021) Optimum sleep is important for reducing cardiovascular risk. The Physician vol 7; Issue 1: 1-6 Art 10 ePub 12.6.21 do https://doi.org/10.38192/1.7.1.10

Article Information Submitted 5.6.21

Published 12.6.21

ISSN 2732-513X (Print)

ISSN 2732-5148 (Online)

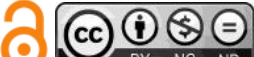

scienceOPEN.com 
Insufficient sleep in teenagers is associated with increased risk of self-harm, suicidal thoughts, and suicide attempts.

In adults there is a link between insufficient sleep and CVD risk. Short sleep duration, including shift-working, has been linked to several factors important for cardiovascular health, including obesity, hypertension, stroke, diabetes and heart attacks [5]. Observational studies have observed cross-sectional associations between short sleep duration (generally $<6 \mathrm{~h}$ per night) and increased body mass index or obesity, prevalent diabetes, and prevalent hypertension. Some studies also reported an association between selfreported long sleep duration (generally $>8 \mathrm{~h}$ per night) and cardiometabolic disease. Prospective studies have found a significant increased risk of weight gain, incident diabetes, and incident hypertension associated with inadequate sleep. [6] In subjects who slept $<6$ hours, risk of CVD, hypertension and stroke was associated with a 1.8 to nearly 3 times higher mortality.[7]

A longitudinal cohort study involving 20,000 people with no CVD at baseline, showed that individuals who slept less than 6 hours per night had a 15\% higher risk of CVD and a $23 \%$ higher risk of coronary heart disease (CHD) compared to people who slept 7-8 hour. Short sleepers with poor sleep quality had a $63 \%$ higher risk of CVD (HR: 1.63 [1.21-2.19]) and a 79\% higher risk of CHD incidence (HR: 1.79 [1.24-2.58]) compared to normal sleepers with good sleep quality, after adjustments for all confounders. [8] Therefore, it is not only duration, but quality of sleep impacts health.

Working atypical shifts is associated with disturbed sleep and insomnia, as well as an increase in CVD risk and mortality. Individuals who manifest shift work sleep disorder (SWSD) usually report trouble sleeping, excessive sleepiness and fatigue which interfere with overall functioning. Misalignment of circadian timing system results in undesirable health consequences. [9]

\section{Proposed Mechanisms}

The pathophysiology is associated with the disruption of normal circadian rhythm. Most physiological processes in the brain and body exhibit daily (circadian) rhythms coordinated by an endogenous clock located in the suprachiasmatic nucleus of the hypothalamus that are essential for normal health and functioning. Exposure to sunlight during the day and darkness at night optimally entrains biological rhythms to promote homeostasis and human health. A major consequence of the modern lifestyle is increased exposure to sun-free environments during the day and artificial lighting at night. Additionally, behavioural disruptions to circadian rhythms (ie, repeated transmeridian flights, night or rotating shift work, or sleep disturbances) have a profound influence on health and have been linked to a number of pathological conditions. [10]

It is hypothesised that a lack of sleep impacts circulating levels of leptin and ghrelin [11]. Leptin is secreted by adipose cells and acts to suppress appetite, whereas ghrelin, a stomach-derived peptide, stimulates appetite. In a sleep deprivation study, short sleep duration was associated with a high body mass index, reduced leptin and increased ghrelin levels [10]. This change in hormone profile leads to the development of obesity via an increase calorie intake and reduction in energy expenditure [12].

Metabolic dysfunction, in the form of insulin resistance and impaired glucose regulation, is an important risk factor for CVD [13] and is associated with disordered sleep. The health risk might be increased in people with a fasting glucose concentration as low as $5.6 \mathrm{mmol} / \mathrm{L}$ or $\mathrm{HbA}_{1 \mathrm{c}}$ of 39 $\mathrm{mmol} / \mathrm{mol}$. Spiegel et al. found that restricting sleep to 4 hours per night for 6 consecutive nights led to a $40 \%$ decrease in glucose tolerance and a reduction in acute insulin response to glucose [14]. Evening cortisol concentrations were raised and activity of the sympathetic nervous system was increased in the sleep-debt condition. In accordance with this, there is an epidemiological association between short sleep duration and an increased incidence of diabetes.[15]

\section{Insomnia and Behavioural Risk Factors}

Insomnia, defined as a difficulty falling or remaining asleep, is associated with increased risk of myocardial infarctions, CHD and CVD mortality $[16,17]$. In addition to the hormonal disbalance (leptin versus ghrelin), insomnia is associated with negative lifestyle choices that increase the risk of CVD. A cohort study of 3000 participants found that individuals with insomnia were more likely to report increased alcohol use, smoking, lack of exercise and impaired cardiopulmonary fitness. [18] The proposed mechanisms are unclear, however, it may be associated with increased psychological and physiological stress leading to adverse cardiovascular consequences.[19]

\section{Obstructive Sleep Apnoea}

Obstructive sleep apnoea (OSA) is a chronic condition characterised by repeated episodes of partial or 
complete collapse of the upper respiratory airway during sleep. The reduction or cessation of airflow (known as hypopnoea and apnoea, respectively) leads to adverse physiological consequences [20]. However, the majority of patients with OSA remain unaware, undiagnosed and untreated for years. [21,22]

OSA is an independent risk factor for hypertension and CVD, and is associated with increased risk of stroke, metabolic syndrome and mortality. A meta-analysis included $3,162,083$ participants. Compared to the control group, the pooled Hazard Ratio of all-cause mortality was 1.19 (95\% Cl, 0.86-1.65) for mild OSA, 1.28 (0.96-1.69) for moderate OSA, and 2.13 (1.682.68) for severe OSA. The pooled HR of cardiovascular mortality was 1.24 (0.53-2.55) for mild OSA, 2.05 (0.575.47) for moderate OSA, and 2.73 (1.94-3.85) for severe OSA. All-cause mortality (HR 0.66; 0.59-0.73) and cardiovascular mortality (HR 0.37; 0.16-0.54) were significantly lower in CPAP-treated than in untreated patients. There were no differences in cardiovascular mortality in CPAP-treated OSA patients vs. normal control subjects (HR 0.82; 0.52-1.29).[23] The physiological injury from intermittent hypoxia, hypercapnoea and intrathoracic pressure changes is thought to be the underlying causal mechanism. These noxious stimuli can, in turn, depress myocardial contractility, activate the sympathetic nervous system, raise blood pressure, heart rate, and myocardial wall stress, depress parasympathetic activity, provoke oxidative stress and systemic inflammation, activate platelets, and impair vascular endothelial function.[24]

\section{Improving Cardiovascular Health Through Better Sleep}

Awareness of the importance of sleep for a healthy heart and encouraging sleep hygiene may be effective strategies for decreasing CVD risk at a population level. Public health strategies should consider disordered sleep as a modifiable lifestyle risk factor, alongside factors such as a lack of exercise, smoking and alcohol. In these cases, exercise promoted increased sleep efficiency and duration regardless of the mode and intensity of activity, especially in populations suffering from disease. As exercise is associated with better sleep, it could be a dual target for interventions [25]. Taking a sleep history and screening for insomnia should be incorporated into primary care consultations, via rapid screening tools, such as the Insomnia Severity Index [26]. The first line recommended treatment of insomnia is cognitive behavioural therapy.

People from ethnic minority backgrounds are more likely to suffer from chronic lack of sleep[11] [5].
Despite the major burden of CVD and stroke among Black and minority ethnic groups in the UK, the majority of the evidence on the management of such conditions has been based on predominantly white European populations. Moreover, the CV epidemiology of African Americans does not represent well the morbidity and mortality experience seen in black Africans and black Caribbean, both in Britain and in their native African countries.

In particular, atherosclerotic disease and coronary heart disease are still relatively rare in the latter groups. This is unlike the South Asian diaspora, who have prevalence rates of CVD in epidemic proportions both in the diaspora and on the subcontinent.[27] Given the enhanced risk of cardiovascular disease, they represent a population who may particularly benefit from primary care interventions to improve cardiovascular health through sleep.

In addition to lifestyle measures, pharmacological interventions to improve sleep represent novel therapeutic targets for managing hypertension. Melatonin, a hormone with a central role in sleep via regulation of circadian rhythms, is a target molecule [28]. Grossman et al. found that 4 weeks of melatonin treatment resulted in a significant decrease in nocturnal blood pressure, compared to placebo.[29]

Treatment of OSA is also an important strategy. The HeartBEAT trial [30] demonstrated that 12 weeks of continuous positive airway pressure (CPAP) therapy led to a significant reduction of 24-hour mean arterial blood pressure in patients with CVD risk factors who were screened for and diagnosed with OSA. This decrease in CVD risk was confirmed in meta-analysis comparing CPAP with other treatments [31]. Therefore, early diagnosis of OSA via screening of patients who have traditional cardiovascular risk factors may be an effective strategy to reduce CVD risk $[32,33]$.

\section{Conclusion}

Impaired or disordered sleep represents an underrecognised risk factor for cardiovascular health. Strategies to improve sleep should be explored through research and public health interventions.

\section{References:}

1 Institute of Medicine (US) Committee on Sleep Medicine and Research. Sleep Disorders and Sleep Deprivation: An Unmet Public Health Problem. Washington (DC): : National Academies 
Press (US) 2006.

http://www.ncbi.nlm.nih.gov/books/NBK19960/

(accessed 12 Jun 2021).

2 Cappuccio FP, D’Elia L, Strazzullo P, et al. Sleep duration and all-cause mortality: a systematic review and meta-analysis of prospective studies. Sleep 2010;33:585-92. doi:10.1093/sleep/33.5.585

3 Watson NF, Badr MS, Belenky G, et al. Recommended Amount of Sleep for a Healthy Adult: A Joint Consensus Statement of the American Academy of Sleep Medicine and Sleep Research Society. Sleep 2015;38:843-4. doi:10.5665/sleep.4716

4 Paruthi S, Brooks LJ, D'Ambrosio C, et al. Consensus Statement of the American Academy of Sleep Medicine on the Recommended Amount of Sleep for Healthy Children: Methodology and Discussion. J Clin Sleep Med 2016;12:1549-61. doi:10.5664/jcsm.6288

5 Grandner MA. Chapter 9 - Obstacles to overcome when improving sleep health at a societal level. In: Grandner MA, ed. Sleep and Health. Academic Press 2019. 107-15. doi:10.1016/B978-0-12-815373-4.00009-5

6 Knutson KL. Does inadequate sleep play a role in vulnerability to obesity? Am J Hum Biol 2012;24:361-71. doi:10.1002/ajhb.22219

7 Fernandez-Mendoza J, He F, Vgontzas AN, et al. Interplay of Objective Sleep Duration and Cardiovascular and Cerebrovascular Diseases on Cause-Specific Mortality. Journal of the American Heart Association 2019;8:e013043. doi:10.1161/JAHA.119.013043

8 Hoevenaar-Blom MP, Spijkerman AMW, Kromhout D, et al. Sleep duration and sleep quality in relation to 12 -year cardiovascular disease incidence: the MORGEN study. Sleep 2011;34:1487-92. doi:10.5665/sleep.1382

9 McFarlane SI. Shift work and sleep: medical implications and management. SMDIJ 2017;1. doi:10.15406/smdij.2017.01.00008

10 Ball LJ, Palesh O, Kriegsfeld LJ. The Pathophysiologic Role of Disrupted Circadian and Neuroendocrine Rhythms in Breast
Carcinogenesis. Endocr Rev 2016;37:450-66.

doi:10.1210/er.2015-1133

11 Grandner MA, Patel NP, Perlis ML, et al. Obesity, diabetes, and exercise associated with sleeprelated complaints in the American population. $Z$ Gesundh Wiss 2011;19:463-74. doi:10.1007/s10389-011-0398-2

12 Spiegel K, Tasali E, Leproult R, et al. Effects of poor and short sleep on glucose metabolism and obesity risk. Nat Rev Endocrinol 2009;5:253-61. doi:10.1038/nrendo.2009.23

13 Huang $\mathrm{Y}$, Cai X, Mai W, et al. Association between prediabetes and risk of cardiovascular disease and all cause mortality: systematic review and meta-analysis. BMJ 2016;355:i5953. doi:10.1136/bmj.i5953

14 Spiegel K, Leproult R, Van Cauter E. Impact of sleep debt on metabolic and endocrine function. Lancet 1999;354:1435-9. doi:10.1016/S01406736(99)01376-8

15 Tasali E, Leproult R, Spiegel K. Reduced sleep duration or quality: relationships with insulin resistance and type 2 diabetes. Prog Cardiovasc Dis 2009;51:381-91.

doi:10.1016/j.pcad.2008.10.002

16 Insomnia and Risk of Cardiovascular Disease CHEST.

https://journal.chestnet.org/article/S00123692(17)30134-4/fulltext (accessed 12 Jun 2021).

17 Phillips Barbara, Mannino David M. Do Insomnia Complaints Cause Hypertension or

Cardiovascular Disease? Journal of Clinical Sleep Medicine 2007;03:489-94. doi:10.5664/jcsm.26913

18 Routledge FS, Dunbar SB, Higgins $M$, et al. Insomnia Symptoms Are Associated With Abnormal Endothelial Function. Journal of Cardiovascular Nursing 2017;32:78-85. doi:10.1097/JCN.0000000000000295

19 Hall MH, Brindle RC, Buysse DJ. Sleep and cardiovascular disease: Emerging opportunities for psychology. Am Psychol 2018;73:994-1006. doi:10.1037/amp0000362 
20 Sleep Apnea and Cardiovascular Disease | Circulation.

https://www.ahajournals.org/doi/10.1161/circul ationaha.107.189420 (accessed 12 Jun 2021).

21 Broström A, Sunnergren O, Årestedt K, et al. Factors associated with undiagnosed obstructive sleep apnoea in hypertensive primary care patients. Scand J Prim Health Care 2012;30:10713. doi:10.3109/02813432.2012.675563

22 Finkel KJ, Searleman AC, Tymkew H, et al. Prevalence of undiagnosed obstructive sleep apnea among adult surgical patients in an academic medical center. Sleep Medicine 2009;10:753-8. doi:10.1016/j.sleep.2008.08.007

23 Fu Y, Xia Y, Yi H, et al. Meta-analysis of all-cause and cardiovascular mortality in obstructive sleep apnea with or without continuous positive airway pressure treatment. Sleep Breath 2017;21:181-9. doi:10.1007/s11325-016-1393-1

24 Obstructive sleep apnoea and its cardiovascular consequences - The Lancet.

https://www.thelancet.com/journals/lancet/arti cle/PIIS0140-6736(08)61622-0/fulltext (accessed 12 Jun 2021).

25 Dolezal BA, Neufeld EV, Boland DM, et al. Interrelationship between Sleep and Exercise: $\mathrm{A}$ Systematic Review. Adv Prev Med 2017;2017:1364387. doi:10.1155/2017/1364387

26 Bastien $\mathrm{CH}$, Vallières $\mathrm{A}$, Morin $\mathrm{CM}$. Validation of the Insomnia Severity Index as an outcome measure for insomnia research. Sleep Med 2001;2:297-307. doi:10.1016/s13899457(00)00065-4

27 Lip GYH, Barnett AH, Bradbury A, et al. Ethnicity and cardiovascular disease prevention in the United Kingdom: a practical approach to management. J Hum Hypertens 2007;21:183211. doi:10.1038/sj.jhh.1002126

28 Tordjman S, Chokron S, Delorme R, et al. Melatonin: Pharmacology, Functions and Therapeutic Benefits. Curr Neuropharmacol 2017; 15:434-43. doi:10.2174/1570159X14666161228122115

29 Grossman E, Laudon M, Yalcin R, et al. Melatonin reduces night blood pressure in patients with nocturnal hypertension. Am J Med
2006;119:898-902.

doi:10.1016/j.amjmed.2006.02.002

30 Gottlieb DJ, Punjabi NM, Mehra R, et al. CPAP versus Oxygen in Obstructive Sleep Apnea. $N$ Engl J Med 2014;370:2276-85. doi:10.1056/NEJMoa1306766

31 Fava C, Dorigoni S, Dalle Vedove F, et al. Effect of CPAP on Blood Pressure in Patients With OSA/Hypopnea. Chest 2014;145:762-71. doi:10.1378/chest.13-1115

32 McNicholas WT. Screening for sleep-disordered breathing: the continuing search for a reliable predictive questionnaire. The Lancet Respiratory Medicine 2016;4:683-5. doi:10.1016/S22132600(16)30119-9

33 Netzer NC, Stoohs RA, Netzer CM, et al. Using the Berlin Questionnaire To Identify Patients at Risk for the Sleep Apnea Syndrome. Ann Intern Med 1999;131:485-91. doi:10.7326/0003-4819131-7-199910050-00002 